\title{
Association study of circulating endothelial progenitor cells and plasma prostacyclin levels in pulmonary hypertension rats
}

\author{
Y. Song \\ Department of Respiratory Medicine, \\ Daping Hospital of the Third Military Medical University, Chongqing, China \\ Corresponding author: Y. Song \\ E-mail: yi_song123@126.com
}

Genet. Mol. Res. 13 (1): 438-444 (2014)

Received August 12, 2013

Accepted October 18, 2013

Published January 21, 2014

DOI http://dx.doi.org/10.4238/2014.January.21.11

\begin{abstract}
The aim of this study was to investigate the correlation between circulating endothelial progenitor cell (EPC) levels and plasma prostacyclin (PGI2) concentrations in pulmonary hypertension $(\mathrm{PH})$ rat models. Monocrotaline was used to induce $\mathrm{PH}$, and the number of CD34/Flk-1-double-positive mononuclear cells in peripheral CD45negative blood was counted by flow cytometry, which reflected EPC circulation. The Greiss method was used to detect the plasma PGI2 concentration. Simple linear regression analysis was used to analyze the relationship between EPC and PGI2. Furthermore, the expressions of cycled EPC, CD34, and Flk-1 were detected under in vitro culture conditions. The mean circulating EPC level in the PH model group $(0.013 \pm 0.004 \%)$ was significantly lower $(\mathrm{P}<0.01)$ than that in the control group $(0.036 \pm 0.009 \%)$. The concentration of plasma PGI2 also significantly decreased $(24.36 \pm 2.08$ vs $58.34 \pm 3.31 \mu \mathrm{M} ; \mathrm{P}<0.01)$. There was a positive correlation between EPC and PGI2 ( $\mathrm{r}=0.870, \mathrm{P}<$ $0.05)$. Under in vitro culture conditions, the EPC number and the CD34 and Flk-1 expression levels in the PH model group were significantly lower than those in the control group. Together, these results suggest
\end{abstract}


that the occurrence of $\mathrm{PH}$ may be associated with decreased plasma PGI2 concentrations, resulting in the reduction of circulating EPC.

Key words: High blood pressure; Pulmonary hypertension; Prostacyclin; Endothelial progenitor cells

\section{INTRODUCTION}

Pulmonary hypertension (PH) is a disease that seriously threatens human health, and is caused by a variety of factors. Its main features include pulmonary vascular structure reconstruction, a sharp decrease in the number and area of the pulmonary vascular bed, a continuous rise in pulmonary vascular resistance, and right ventricular hypertrophy, and the prognosis is often poor. In recent years, a large number of studies have shown that endothelial progenitor cells (EPCs) play a crucial role in vascular injury repair. The peripheral EPCs in PH patients show some abnormalities in terms of their number and function, suggesting that EPCs may be an important factor in the pathogenesis of PH. Furthermore, decreased levels of plasma prostacyclin (PGI2) will not only lead to the dysfunction of vasodilation, but can also significantly affect EPC mobilization and homing. Therefore, in this study, subcutaneous injection of monocrotaline was used to induce experimental PH, and then the plasma PGI2 and EPC levels in the circulatory system were detected to investigate the association between these two factors in the $\mathrm{PH}$ process.

\section{MATERIAL AND METHODS}

\section{Material}

The main reagents used in the experiments were as follows: monocrotaline (Sigma Company), PE-labeled goat anti-rat CD34, fluorescein isothiocyanate (FITC)-labeled goat anti-rat Flk-1, APC-labeled goat anti-rat CD45 (BD Company), the PGI2 detection kit (Sigma Chemical Co.), EGM-2 (Lonza Co.), DIL-labeled acetylated low-density lipoprotein (DILacLDL; Molecular Probes Co.), FITC-labeled hemagglutinin (FITC-lectin; Sigma Co.), and fibronectin (Hematological Technologies Co.).

\section{Animal model establishment and grouping}

Sixteen healthy 8-week-old male Sprague-Dawley rats were selected, ranging in weight from 150 to $200 \mathrm{~g}$. They were randomly divided into two groups, with 8 rats in each group. After 2 weeks of adaptability feeding, rats in the model group received subcutaneous injections of $50 \mathrm{mg} / \mathrm{kg} \mathrm{1 \%}$ monocrotaline; the PH model would be formed 3 weeks after injection (Matsuda et al., 2005). Rats in the control group received an equal volume of phosphatebuffered saline (PBS) via subcutaneous injection.

\section{Circulating EPC determination}

Three weeks after injection, peripheral blood samples in rats of the model and control 
groups were collected from the tail vein and placed in ethylenediaminetetraacetic acid (EDTA) anticoagulant tubes. Circulating EPC was defined as CD45-negative, CD34/Flk-1-double-positive mononuclear cells. PE-labeled goat anti-rat CD34, FITC-labeled goat anti-rat Flk-1, and APC-labeled goat anti-rat CD45 were mixed with the collected blood samples for 15 min, and after erythrocyte lysis, flow cytometry was applied to detect the proportion of EPCs in each sample of 100,000 mononuclear cells.

\section{Plasma PGI2 concentration detection}

Peripheral blood samples collected via the tail vein were placed in EDTA anticoagulant tubes and centrifuged to obtain the plasma. The PGI2 detection kit was used to detect the plasma PGI2 concentration according to the Greiss method. All operating procedures were in strict accordance with manufacturer instructions.

\section{Circulating EPC culture in vitro}

Mononuclear cells were obtained from the separated lymphatic fluid of the peripheral blood collected via the tail vein using density gradient centrifugation. The cells were added to EGM-2 medium containing 5\% fetal bovine serum, seeded at a density of $2 \times 10^{6} / \mathrm{mL}$ on $24-$ well plates with fibronectin coating, and incubated at $37^{\circ} \mathrm{C}$ and $5 \% \mathrm{CO}_{2}$. On the 3 rd day, PBS was used to wash away any non-adherent cells and the culture medium was replaced. Half of the medium was changed every other day for the remainder of the experiment.

\section{Immunofluorescence detection}

Seven days after the cell culture, cells were incubated in the EGM-2 medium containing $5 \mathrm{mg} / \mathrm{L}$ DIL-acLDL at $37^{\circ} \mathrm{C}$ for $4 \mathrm{~h}$ and then fixed for $20 \mathrm{~min}$ with $2 \%$ paraformaldehyde cells. After PBS immersion, fixed cells were incubated in PBS containing $5 \mathrm{mg} / \mathrm{L}$ FITC-lectin at $37^{\circ} \mathrm{C}$ for $1 \mathrm{~h}$. Cells were mounted with $90 \%$ neutral glycerol for observation under a confocal microscope. Red fluorescence substances indicated the DIL-acLDL-positive cells, while green fluorescence substances indicated the FITC-lectin-positive cells. Double-fluorescentpositive cells were considered to be EPCs.

\section{Flow cytometry detection}

On the 14th day of the cell culture, single-cell suspensions were prepared, which were stained with PE-CD34 and FITC-Flk-1, along with their corresponding controls, according to manufacturer instructions, and the proportion of various types of cells were detected by flow cytometry. Cells were detected based on the same type of controls.

\section{Statistical analysis}

The SPSS 10.0 statistical package was used for statistical analyses. Data are reported as means \pm standard deviations (SD). The mean EPC content and plasma PGI2 concentration were compared between the two groups using the Student $t$-test, and $\mathrm{P}<0.05$ indicated a sta- 
tistically significant difference. The association between the EPC content and the plasma PGI2 concentration was evaluated by simple linear regression analysis.

\section{RESULTS}

\section{Pulmonary lesions in the rat models}

Compared with rats in the control group, rats in the PH model group showed pulmonary vascular endothelial cell injury, swelling, and necrosis. Platelets adhered to the damaged endothelial cells and the bare basement membrane, and lymphocyte infiltration was evident. The pulmonary artery wall was significantly thickened, while stenosis, occlusion, and muscularized artery hypertrophy were obvious (Figure 1).

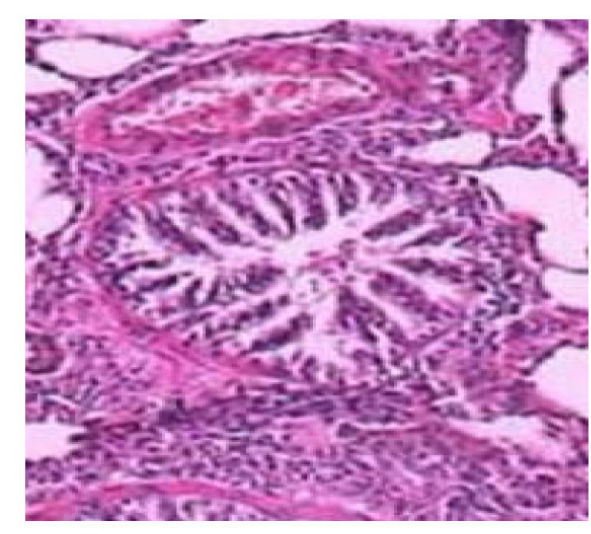

A

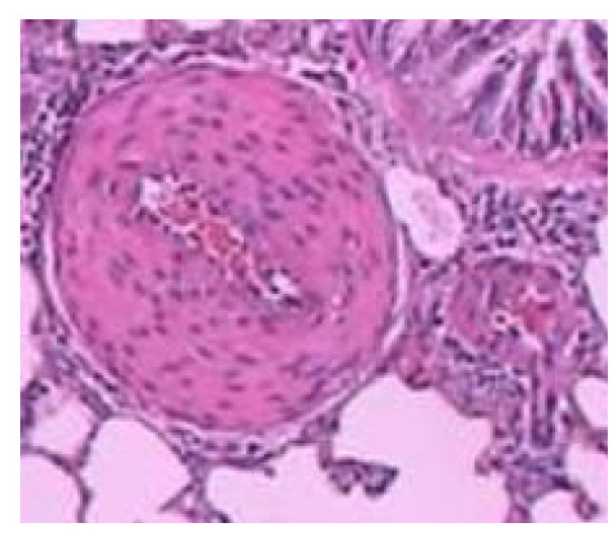

B

Figure 1. Pathology of lung tissue in the groups (HE, 200X). A. Control. B. Pulmonary hypertension model.

\section{Comparison of circulating EPC levels}

Flow cytometry revealed that the mean proportion of circulating EPC in peripheral blood mononuclear cells in the rats of the PH model group $(0.013 \pm 0.004 \%)$ was significantly lower $(\mathrm{P}<0.01)$ than that of the control group $(0.036 \pm 0.009 \%)$.

\section{Plasma PGI2 concentration comparison}

The mean plasma PGI2 concentration of rats in the PH model group $(24.36 \pm 2.08$ $\mu \mathrm{M})$ was significantly lower $(\mathrm{P}<0.01)$ than that of the control group $(58.34 \pm 3.31 \mu \mathrm{M})$.

\section{Correlation between circulating EPC levels and plasma PGI2 concentrations}

Circulating EPC levels and plasma PGI2 concentrations were positively correlated in the PH model group $(\mathrm{r}=0.890, \mathrm{P}<0.05)$ (Figure 2). 


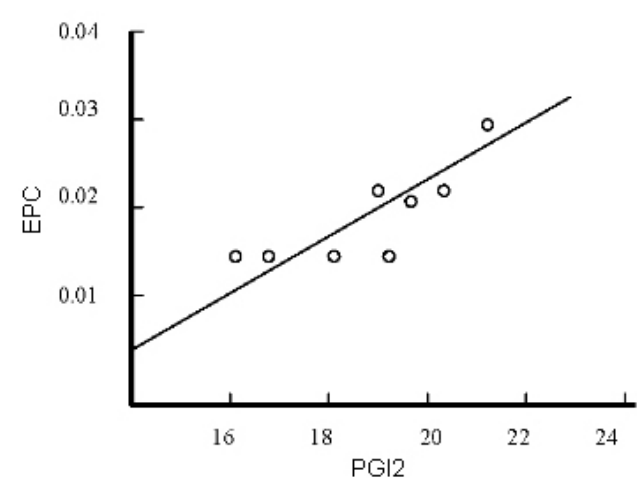

Figure 2. Relationship between endothelial progenitor cells (EPC) and PGI2.

\section{EPC growth in vitro}

The cellular aggregation could be seen 3 days after the peripheral blood mononuclear cell culture. They presented colony-like growth, similar blood island-like structures, and radial distribution of the spindle cells was visible (Figure 3A); spindle cells increased with prolonged incubation. Seven days after induction, the EPCs generated from the cell cultures showed a tendency to combine with the hemagglutinin (Figure 3B) and engulfed the acetylated low-density lipoprotein (Figure 3C). The proportion of double-positive cells (Figure 3D) in the PH model group was slightly, but significantly, lower than that in the normal group ( $\mathrm{P}$ $<0.05)$. At the 14th day of cell culture, flow cytometry revealed that the mean proportion of CD34-positive cells in the experimental group was $14.25 \pm 3.21 \%$, while the mean proportion of Flk-1-positive cells was $38.52 \pm 3.81 \%$. In the control group, the mean proportion of CD34positive cells was $23.11 \pm 3.02 \%$, while the mean proportion of Flk-1-positive cells was 46.6 $\pm 3.15 \%$. Proportions of both CD34 and Flk-1-positive cells were significantly lower in the experimental group than in the normal control group $(\mathrm{P}<0.01)$.
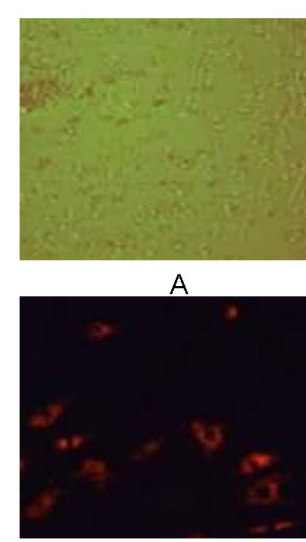

C

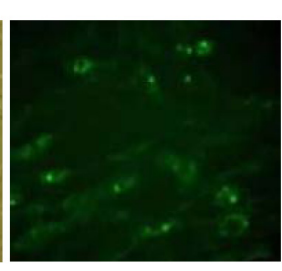

B

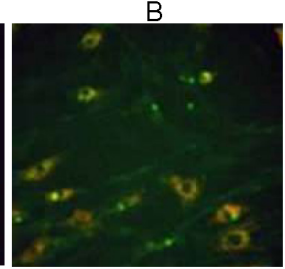

D

Figure 3. Bone marrow growth and identification of the endothelial progenitor cells. A. After 7 days of culture, similar blood island-like structures and visible radially distributed spindle cells are seen; B. cell binding hemagglutinin shows green fluorescent; C. phagocytosis of acetylated low-density lipoprotein shows red fluorescence; D. doublepositive cells shows yellow fluorescence (100X). 


\section{DISCUSSION}

PH is caused by persistent vasospasm or vascular muscle hyperplasia, which will ultimately lead to pulmonary vascular remodeling. Determining how to stop and reverse pulmonary vascular remodeling is the key for effective prevention and treatment of PH. Conventional vasodilator therapy can alleviate vasospasm in early onset $\mathrm{PH}$, but it is useless in the late stage of vascular remodeling. Therefore, the fundamental solution to $\mathrm{PH}$ pathology may lie in methods to prevent and reverse pulmonary vascular remodeling and to expand the cross-sectional area of the vascular bed as soon as possible. In recent years, numerous studies have shown that EPCs play a crucial role in angiogenesis and in the maintenance of normal endothelial function and repair after vascular endothelial cell damage. Under in vitro culture conditions, EPCs can also be differentiated into mature endothelial cells, which have normal phenotypes and function. EPCs can be integrated into the ischemic damaged areas in experimental animals to generate the capillaries and to improve perfusion of the ischemic region (Asahara et al., 1997; Kalka et al., 2000; Fan et al., 2005). Indeed, studies have shown that the peripheral blood EPC number increased significantly during the acute PH stage (Marsboom et al., 2008), suggesting that when pulmonary endothelial injury occurs, EPC mobilization and homing in the body would occur automatically in order to achieve restoration of vascular injury and to maximize the recovery of normal morphology and function. By contrast, in cases of irreversible vascular remodeling, the peripheral blood EPC number and activity were significantly reduced (Diller et al., 2008; Junhui et al., 2008). Through early exogenous EPC implants, monocrotaline-induced PH model lesions could be effectively prevented (Yip et al., 2008). In the process of PH, either EPCs or the premise cells showed a reduction in number or functional defects, which prevented EPCs from carrying out their role in the repair of vascular injury. Alternatively, the persistent lack of blood stimulating factors to mobilize bone marrow proliferation and homing of EPC might be the main mechanism; however, this has been rarely studied. Some studies have shown that prostacyclin synthase is involved in EPC mobilization, homing, and differentiation. Prostacyclin synthase gene knockout mice had obvious EPC mobilization defects (He et al., 2004), and repair of the damaged artery was also inhibited (Laufs et al., 2004). These results indicated that the plasma PGI2 played an important role as an EPC-stimulating factor during the vascular injury repair process. In the present study, monocrotaline was used to successfully induce the PH models. Levels of circulating EPCs and the concentration of plasma PGI2 were also detected. Results showed that circulating EPC levels and plasma PGI2 were reduced in PH models compared with those in the control group, and the two factors were significantly positively correlated. Furthermore, even under suitable culture conditions in vitro, the peripheral blood-derived circulating EPC number, proliferation, differentiation capacity, and the specific surface marker expression in $\mathrm{PH}$ rat models were also significantly reduced. Thus, the development of $\mathrm{PH}$ was indeed correlated with a reduced concentration of plasma PGI2 and a reduction in the number of circulating EPCs.

Since PH lesions after implantation of exogenous EPCs can be effectively controlled (Junhui et al., 2008), the exogenous EPC was not suppressed in the host body, and its normal functioning was not affected. We inferred that during the processes of PH, EPC injury caused by the abnormal immune response may either have not occurred, or the effects of EPC damage were minor in this immune dysfunction. Together with previous research, the results of this study suggest that the fundamental cause of PH may be related to the long time and overloaded work required of bone marrow EPCs to repair vascular injury, the consumption of EPC mo- 
bilization factors, such as blood prostacyclin synthase, and the reduction in the number and function of EPCs.

\section{REFERENCES}

Asahara T, Murohara T, Sullivan A, Silver M, et al. (1997). Isolation of putative progenitor endothelial cells for angiogenesis. Science 275: 964-967.

Diller GP, van Eijl S, Okonko DO, Howard LS, et al. (2008). Circulating endothelial progenitor cells in patients with Eisenmenger syndrome and idiopathic pulmonary arterial hypertension. Circulation 117: 3020-3030.

Fan CL, Gao PJ, Che ZQ, Liu JJ, et al. (2005). Therapeutic neovascularization by autologous transplantation with expanded endothelial progenitor cells from peripheral blood into ischemic hind limbs. Acta Pharmacol. Sin. 26: 1069-1075.

He T, Smith LA, Harrington S, Nath KA, et al. (2004). Transplantation of circulating endothelial progenitor cells restores endothelial function of denuded rabbit carotid arteries. Stroke 35: 2378-2384.

Junhui Z, Xingxiang W, Guosheng F, Yunpeng S, et al. (2008). Reduced number and activity of circulating endothelial progenitor cells in patients with idiopathic pulmonary arterial hypertension. Respir. Med. 102: 1073-1079.

Kalka C, Masuda H, Takahashi T, Kalka-Moll WM, et al. (2000). Transplantation of ex vivo expanded endothelial progenitor cells for therapeutic neovascularization. Proc. Natl. Acad. Sci. U. S. A. 97: 3422-3427.

Laufs U, Werner N, Link A, Endres M, et al. (2004). Physical training increases endothelial progenitor cells, inhibits neointima formation, and enhances angiogenesis. Circulation 109: 220-226.

Marsboom G, Pokreisz P, Gheysens O, Vermeersch P, et al. (2008). Sustained endothelial progenitor cell dysfunction after chronic hypoxia-induced pulmonary hypertension. Stem. Cells 26: 1017-1026.

Matsuda Y, Hoshikawa Y, Ameshima S, Suzuki S, et al. (2005). Effects of peroxisome proliferator-activated receptor gamma ligands on monocrotaline-induced pulmonary hypertension in rats. Nihon Kokyuki. Gakkai Zasshi 43: 283-288.

Yip HK, Chang LT, Sun CK, Sheu JJ, et al. (2008). Autologous transplantation of bone marrow-derived endothelial progenitor cells attenuates monocrotaline-induced pulmonary arterial hypertension in rats. Crit. Care Med. 36: 873-880. 